\title{
Corrigendum
}

\section{Transgelin as a suppressor is associated with poor prognosis in colorectal carcinoma patients}

Liang Zhao, Hui Wang, Yong-Jian Deng, Shuang Wang, Chao Liu, He Jin and Yan-Qing Ding Modern Pathology (2010) 23, 157; doi:10.1038/modpathol.2009.144

Correction to: Modern Pathology (2009) 22, 786-796; doi:10.1038/modpathol.2009.29; published online 27 March 2009
In this article, the authors have replaced Figure 3 with the following revised version:

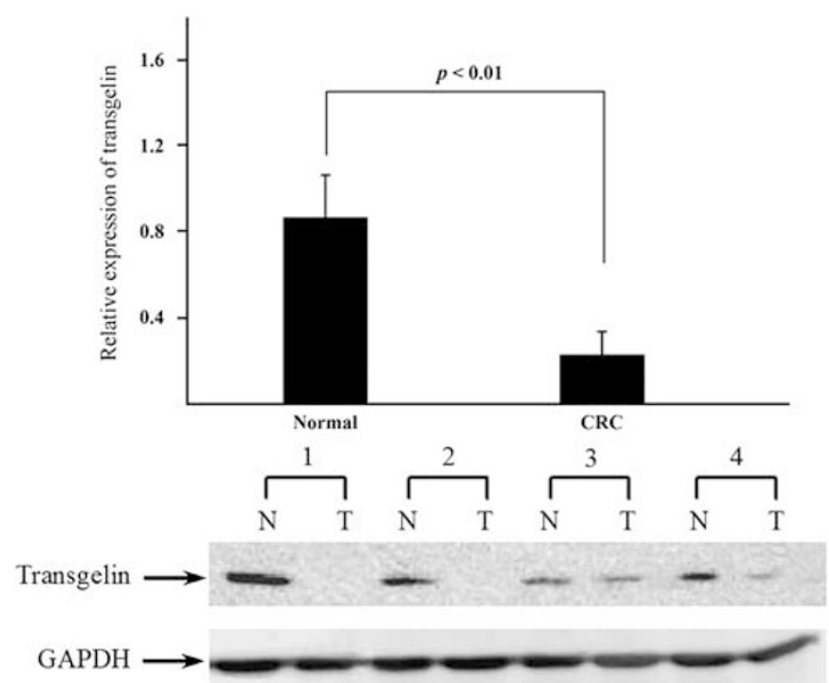

Figure 3 Expression of transgelin in normal colorectal mucosa and primary colorectal cancer samples. There are four representative samples in each group. Immunosignals were quantified by densitometric scanning. Transgelin expression in the individual tissue samples was calculated as transgelin expression relative to GAPDH expression. Data are means \pm s.d. from three independent experiments. Immunoblotting analysis revealed the variation to be consistent with its appearance in the gel images (Figure 2). 